1 Hacettepe Journal of Mathematics and Statistics

$\bigcap$ Volume 45 (5) (2016), 1475-1484

\title{
Integral type contraction and coupled coincidence fixed point theorems for two pairs in G-metric spaces
}

\author{
Akbar Zada*, Rahim Shah ${ }^{\dagger}$ and Tongxing $\mathrm{Li}^{\ddagger}$
}

\begin{abstract}
In this paper, we introduces the idea of integral type contraction with respect to G-metric space and by using the notion of integral type contraction we prove some coupled coincidence fixed point results for two pairs of mapping in G-metric space. Also we give an example as an application point of view.
\end{abstract}

Keywords: G-metric space; couple coincidence point; common fixed point; integral type contraction.

2000 AMS Classification: 47H09; 47H10.

Received : 12.08.2015 Accepted : 04.11.2015 Doi : 15672/HJMS.20164514280

\section{Introduction}

The study of common fixed points of mappings which satisfies certain contractive conditions has been studied by a lot of researchers due to its applications in mathematics. For the study of coincidence point of theory in metric and cone metric spaces we recommend [1, 2, 3, 4, 7, 8, 9, 10, 11, 15, 17, 18]. In 2006 Mustafa and Sims [16], introduced the idea of G-metric space and presented some fixed point theorems in G-metric space. The concept of a coupled coincidence point of mapping was introduced by V. Lakshmikantham $[5,13]$, they also studied some fixed point theorems in partially ordered metric spaces. In 2010 Shatanawi [19] gave the proof of coupled coincidence fixed point theorems in generalized metric spaces. Also in 2014 Manish Kumar [14] proved a coupled coincidence fixed point theorem in the setting of two pairs of mapping in G-metric space. Moreover

\footnotetext{
*Department of Mathematics, University of Peshawar, Peshawar, Pakistan. Email : zadababo@yahoo.com, akbarzada@upesh.edu.pk

$\dagger$ Department of Mathematics, University of Peshawar, Peshawar, Pakistan. Email : safeer_rahim@yahoo.com

${ }^{\ddagger}$ LinDa Institute of Shandong Provincial Key Laboratory of Network Based Intelligent Computing, Linyi University, Linyi, Shandong 276005, P. R. China. School of Informatics, Linyi University, Linyi, Shandong 276005, P. R. China.

Email : litongx2007@163.com
} 
in 2002, Branciari [6] gave the idea of integral type contractive mappings in complete metric spaces and they studied the existence of fixed points for mappings which is defined on complete metric space satisfying integral type contraction. Recently F. Khojasteh et al.[12], gave the idea of integral type contraction in cone metric spaces and proved some fixed point theorems in such spaces. So by using the concept of Branciari [6] of integral type contractive mapping, we presented a coupled coincidence fixed point results of integral type contractive mappings for two pairs in the setting of G-metric spaces. Also we give suitable example that support our main result.

\section{Preliminaries}

We will need the following definitions and results in this paper.

2.1. Definition. [16] Let $Y$ be a non-empty set and $G: Y \times Y \times Y \rightarrow R^{+}$is a function that satisfies the following conditions:

(1) $G(a, b, c)=0$ if $a=b=c$,

(2) $G(a, a, b)>0$ for all $a, b \in Y$ with $a \neq b$,

(3) $G(a, a, b) \leq G(a, b, c)$, for all $a, b, c \in Y$ with $c \neq b$

(4) $G(a, b, c)=G(a, c, b)=G(b, c, a)=\ldots$, symmetry in all variables,

(5) $G(a, b, c) \leq G(a, s, s)+G(s, b, c)$ for all $a, b, c, s \in Y$.

Then the function $G$ is called a generalized metric and the pair $(Y, G)$ is called a G-metric space.

2.2. Example. [16] Let $Y=\{x, y\}$. Define $G$ on $Y \times Y \times Y$ by

$$
G(x, x, x)=G(y, y, y)=0, G(x, x, y)=1, G(x, y, y)=2
$$

and extend $\mathrm{G}$ to $Y \times Y \times Y$ by using the symmetry in the variables. Then it is clear that $(Y, G)$ is a $\mathrm{G}$-metric space.

2.3. Definition. [16] Let $(Y, G)$ be a G-metric space and $\left(a_{n}\right)$ a sequence of points of $Y$. A point $a \in Y$ is said to be the limit of the sequence $\left(a_{n}\right)$, if $\lim _{n, m \rightarrow+\infty} G\left(a, a_{n}, a_{m}\right)=0$ and we say that the sequence $\left(a_{n}\right)$ is G-convergent to $a$.

2.1. Proposition. [16] Let $(Y, G)$ be a G-metric space. Then the following are equivalent:

(1) $\left(a_{n}\right)$ is G-convergent to $a$.

(2) $G\left(a_{n}, a_{n}, a\right) \rightarrow 0$ as $n \rightarrow+\infty$.

(3) $G\left(a_{n}, a, a\right) \rightarrow 0$ as $n \rightarrow+\infty$.

(4) $G\left(a_{n}, a_{m}, a\right) \rightarrow 0$ as $n, m \rightarrow+\infty$

2.4. Definition. [15] Let $(Y, G)$ be a G-metric space. A sequence $\left(a_{n}\right)$ is called GCauchy if for every $\epsilon>0$, there is $k \in \mathbf{N}$ such that $G\left(a_{n}, a_{m}, a_{l}\right)<\epsilon$, for all $n, m, l \geq k$; that is $G\left(a_{n}, a_{m}, a_{l}\right) \rightarrow 0$ as $n, m, l \rightarrow+\infty$.

2.2. Proposition. [16] Let $(Y, G)$ be a G-metric space. Then the following are equivalent:

(1) The sequence $\left(a_{n}\right)$ is G-Cauchy.

(2) For every $\epsilon>0$, there is $k \in \mathbf{N}$ such that $G\left(a_{n}, a_{m}, a_{m}\right)<\epsilon$, for all $n, m \geq k$.

2.5. Definition. [16] A G-metric space $(Y, G)$ is called G-complete if every G-Cauchy sequence in $(Y, G)$ is $\mathrm{G}$-convergent in $(Y, G)$.

2.6. Definition. [5] An element $(a, b) \in Y \times Y$ is called a coupled coincidence point of the mappings $F: Y \times Y \rightarrow Y$ and $g: Y \rightarrow Y$ if $F(a, b)=g a$ and $F(b, a)=g b$. 
2.7. Definition. [13] Let $\mathrm{Y}$ be a non-empty set. Then we say that the mappings $F$ : $Y \times Y \rightarrow Y$ and $g: Y \rightarrow Y$ are commutative if $g F(a, b)=F(g a, g b)$.

2.8. Definition. [13] An element $(a, b) \in Y \times Y$ is called a coupled fixed point of mapping $F: Y \times Y \rightarrow Y$ if $F(a, b)=a$ and $F(b, a)=b$.

In 2002, Branciari in [6] introduced a general contractive condition of integral type as follows.

2.9. Theorem. [6] Let $(Y, d)$ be a complete metric space, $\alpha \in(0,1)$, and $f: Y \rightarrow Y$ is a mapping such that for all $x, y \in Y$,

$$
\int_{0}^{d(f(x), f(y))} \phi(t) d t \leq \alpha \int_{0}^{d(x, y)} \phi(t) d t
$$

where $\phi:[0,+\infty) \rightarrow[0,+\infty)$ is Lebesgue-integrable mapping which is summable (i.e., with finite integral ) on each compact subset of $[0,+\infty)$ such that for each $\epsilon>0$, $\int_{0}^{\epsilon} \phi(t) d t>0$, then $f$ has a unique fixed point $a \in Y$, such that for each $x \in Y$, $\lim _{n \rightarrow \infty} f^{n}(x)=a$.

We use the above idea of Branciari [6] and presented some coupled coincidence fixed point results of integral type contraction in G-metric space.

\section{Main Results}

In this section we will prove some fixed point results for two pairs in G-metric space by using integral type contractive mapping. We will start our work with the following important lemma.

3.1. Lemma. Let $(Y, G)$ be a G-metric space. Suppose $F, S: Y \times Y \rightarrow Y$ and $g, h$ : $Y \rightarrow Y$ be two mappings such that

$$
\int_{0}^{G(F(a, b), S(p, q), S(c, r))} \varphi(t) d t \leq k \int_{0}^{(G(h a, g p, g c)+G(h b, g q, g r))} \varphi(t) d t
$$

for all $a, b, c, p, q, r \in Y$ and $\varphi:[0,+\infty) \rightarrow[0,+\infty)$ is a Lebesgue integrable mapping which is summable such that for each $\epsilon>0, \int_{0}^{\epsilon} \varphi(t) d t>0$. Assume that $(a, b)$ is coupled coincidence point of the pairs of mappings $\{F, h\}$ and $\{S, g\}$ and $g a=h a$ and $g b=h b$. If $k \in\left[0, \frac{1}{8}\right)$, then

$S(a, b)=g a=g b=S(b, a)$ and $F(a, b)=h a=h b=F(b, a)$.

Proof. Since $(a, b)$ is a coupled coincidence point of the mappings $\{F, h\}$ and $\{S, g\}$, we have $h a=F(a, b), h b=F(b, a)$ and $g a=S(b, a), g b=S(b, a)$. Suppose $g a \neq g b$. Then by (3.1), we get

$$
\begin{aligned}
\int_{0}^{G(g a, g b, g b)} \varphi(t) d t & =\int_{0}^{G(F(a, b), S(b, a), S(b, a))} \varphi(t) d t \\
& \leq k \int_{0}^{(G(h a, g b, g b)+G(h b, g a, g a))} \varphi(t) d t \\
& =k \int_{0}^{(G(g a, g b, g b)+G(g b, g a, g a))} \varphi(t) d t
\end{aligned}
$$


Also we have,

$$
\begin{aligned}
\int_{0}^{G(g b, g a, g a)} \varphi(t) d t & =\int_{0}^{G(F(b, a), S(a, b), S(a, b))} \varphi(t) d t \\
& \leq k \int_{0}^{(G(h b, g a, g a)+G(h a, g b, g b))} \varphi(t) d t \\
& =k \int_{0}^{(G(g b, g a, g a)+G(g a, g b, g b))} \varphi(t) d t .
\end{aligned}
$$

Therefore

$$
\int_{0}^{G(g a, g b, g b)} \varphi(t) d t+\int_{0}^{G(g b, g a, g a)} \varphi(t) d t \leq 2 k \int_{0}^{(G(g a, g b, g b)+G(g b, g a, g a))} \varphi(t) d t .
$$

Since $2 k<1$, we get

$$
\int_{0}^{G(g a, g b, g b)} \varphi(t) d t+\int_{0}^{G(g b, g a, g a)} \varphi(t) d t<\int_{0}^{G(g a, g b, g b)} \varphi(t) d t+\int_{0}^{G(g b, g a, g a)} \varphi(t) d t
$$

which is a contradiction. So $g a=g b$, and hence

$$
S(a, b)=g a=g b=S(b, a) \text { and } F(a, b)=h a=h b=F(b, a) .
$$

3.1. Theorem. Let $(Y, G)$ be a G-metric space. Let $F, S: Y \times Y \rightarrow Y$ and $g, h: Y \rightarrow Y$ be two mappings such that

$$
\int_{0}^{G(F(a, b), S(p, q), S(c, r))} \varphi(t) d t \leq k \int_{0}^{(G(h a, g p, g c)+G(h b, g q, g r))} \varphi(t) d t
$$

for all $a, b, c, p, q, r \in Y$ and $\varphi:[0,+\infty) \rightarrow[0,+\infty)$ is a Lebesgue integrable mapping which is summable such that for each $\epsilon>0, \int_{0}^{\epsilon} \varphi(t) d t>0$. Assume that $F, S$ and $g, h$ satisfy the following conditions:

(i) $F(Y \times Y) \subset g(Y)$ and $S(Y \times Y) \subset h(Y)$

(ii) $g(Y)$ or $h(Y)$ is complete and

(iii) $g$ and $h$ are G-continuous and pairs $\{F, h\}$ and $\{S, g\}$ are commuting mappings.

If $k \in\left[0, \frac{1}{8}\right)$, then there is a unique $a \in Y$ such that $F(a, a)=S(a, a)=g(a)=h(a)=a$.

Proof. Let $a_{0}, b_{0} \in Y$. Since $F(Y \times Y) \subset g(Y)$, choose $a_{1}, b_{1} \in Y$ such that $u_{1}^{\prime}=$ $g a_{1}=F\left(a_{0}, b_{0}\right)$ and $v_{1}^{\prime}=g b_{1}=F\left(b_{0}, a_{0}\right)$. Again since $S(Y \times Y) \subset h(Y)$, choose $a_{2}, b_{2} \in Y$ such that $u_{2}^{\prime}=h a_{2}=S\left(a_{1}, b_{1}\right)$ and $v_{2}^{\prime}=h b_{2}=S\left(b_{1}, a_{1}\right)$. Continuing this process, we can construct two sequences $\left(u_{n}^{\prime}\right)$ and $\left(v_{n}^{\prime}\right)$ in Y such that $u_{2 n+1}^{\prime}=g a_{2 n+1}=$ $F\left(a_{2 n}, b_{2 n}\right), v_{2 n}^{\prime}=g b_{2 n+1}=F\left(b_{2 n+1}, a_{2 n+1}\right)$ and $u_{2 n+2}^{\prime}=h a_{2 n+2}=S\left(a_{2 n+1}, b_{2 n+1}\right)$, $v_{2 n+2}^{\prime}=h b_{2 n+2}=S\left(b_{2 n+1}, a_{2 n+1}\right)$. For $n \in N$, we have

$$
\begin{aligned}
\int_{0}^{G\left(u_{2 n+1}^{\prime}, u_{2 n+2}^{\prime}, u_{2 n+2}^{\prime}\right)} \varphi(t) d t & =\int_{0}^{\left(G\left(F\left(a_{2 n}, b_{2 n}\right), S\left(a_{2 n+1}, b_{2 n+1}\right), S\left(a_{2 n+1}, b_{2 n+1}\right)\right)\right.} \varphi(t) d t \\
& \leq k \int_{0}^{\left(G\left(h a_{2 n}, g a_{2 n+1}, g a_{2 n+1}\right)+G\left(h b_{2 n}, g b_{2 n+1}, g b_{2 n+1}\right)\right)} \varphi(t) d t \\
& =k \int_{0}^{\left(G\left(u_{2 n}^{\prime}, u_{2 n+1}^{\prime}, u_{2 n+1}^{\prime}\right)+G\left(v_{2 n}^{\prime}, v_{2 n+1}^{\prime}, v_{2 n+1}^{\prime}\right)\right)} \varphi(t) d t .
\end{aligned}
$$

In the same manner

$$
\int_{0}^{G\left(v_{2 n+1}^{\prime}, v_{2 n+2}^{\prime}, v_{2 n+2}^{\prime}\right)} \varphi(t) d t \leq k \int_{0}^{\left(G\left(v_{2 n}^{\prime}, v_{2 n+1}^{\prime}, v_{2 n+1}^{\prime}\right)+G\left(u_{2 n}^{\prime}, u_{2 n+1}^{\prime}, u_{2 n+1}^{\prime}\right)\right)} \varphi(t) d t .
$$


We have

$$
\begin{aligned}
\int_{0}^{G\left(u_{2 n+1}^{\prime}, u_{2 n+2}^{\prime}, u_{2 n+2}^{\prime}\right)} \varphi(t) d t & +\int_{0}^{G\left(v_{2 n+1}^{\prime}, v_{2 n+2}^{\prime}, v_{2 n+2}^{\prime}\right)} \varphi(t) d t \\
& \leq 2 k \int_{0}^{\left\{G\left(u_{2 n}^{\prime}, u_{2 n+1}^{\prime}, u_{2 n+1}^{\prime}\right)+\left(G\left(v_{2 n}^{\prime}, v_{2 n+1}^{\prime}, v_{2 n+1}^{\prime}\right)\right\}\right.} \varphi(t) d t \\
& \leq 8 k \int_{0}^{\left\{G\left(u_{2 n}^{\prime}, u_{2 n+1}^{\prime}, u_{2 n+1}^{\prime}\right)+\left(G\left(v_{2 n}^{\prime}, v_{2 n+1}^{\prime}, v_{2 n+1}^{\prime}\right)\right\}\right.} \varphi(t) d t,(3.5)
\end{aligned}
$$

holds for all $n \in N$, again from

$$
\begin{aligned}
\int_{0}^{G\left(u_{2 n}^{\prime}, u_{2 n+1}^{\prime}, u_{2 n+1}^{\prime}\right)} \varphi(t) d t & \leq 2 \int_{0}^{G\left(u_{2 n+1}^{\prime}, u_{2 n}^{\prime}, u_{2 n}^{\prime}\right)} \varphi(t) d t \\
& =2 \int_{0}^{\left(G\left(F\left(a_{2 n}, b_{2 n}\right), S\left(a_{2 n-1}, b_{2 n-1}\right), S\left(a_{2 n-1}, b_{2 n-1}\right)\right)\right.} \varphi(t) d t \\
& \leq 2 k \int_{0}^{\left(G\left(h a_{2 n}, g a_{2 n-1}, g a_{2 n-1}\right)+G\left(h b_{2 n}, g b_{2 n-1}, g b_{2 n-1}\right)\right)} \varphi(t) d t \\
& =2 k \int_{0}^{\left(G\left(u_{2 n}^{\prime}, u_{2 n-1}^{\prime}, u_{2 n-1}^{\prime}\right)+G\left(v_{2 n}^{\prime}, v_{2 n-1}^{\prime}, v_{2 n-1}^{\prime}\right)\right)} \varphi(t) d t \\
& \leq 4 k \int_{0}^{\left(G\left(u_{2 n-1}^{\prime}, u_{2 n}^{\prime}, u_{2 n}^{\prime}\right)+G\left(v_{2 n-1}^{\prime}, v_{2 n}^{\prime}, v_{2 n}^{\prime}\right)\right)} \varphi(t) d t \quad(3.6)
\end{aligned}
$$

and

$$
\begin{aligned}
\int_{0}^{G\left(v_{2 n}^{\prime}, v_{2 n+1}^{\prime}, v_{2 n+1}^{\prime}\right)} \varphi(t) d t & \leq 2 \int_{0}^{G\left(v_{2 n+1}^{\prime}, v_{2 n}^{\prime}, v_{2 n}^{\prime}\right)} \varphi(t) d t \\
& =2 \int_{0}^{\left(G\left(F\left(b_{2 n}, a_{2 n}\right), S\left(b_{2 n-1}, a_{2 n-1}\right), S\left(b_{2 n-1}, a_{2 n-1}\right)\right)\right.} \varphi(t) d t \\
& \leq 2 k \int_{0}^{\left(G\left(h b_{2 n}, g b_{2 n-1}, g b_{2 n-1}\right)+G\left(h a_{2 n}, g a_{2 n-1}, g a_{2 n-1}\right)\right)} \varphi(t) d t \\
& =2 k \int_{0}^{\left(G\left(v_{2 n}^{\prime}, v_{2 n-1}^{\prime}, v_{2 n-1}^{\prime}\right)+G\left(u_{2 n}^{\prime}, u_{2 n-1}^{\prime}, u_{2 n-1}^{\prime}\right)\right)} \varphi(t) d t \\
& \leq 4 k \int_{0}^{\left(G\left(u_{2 n-1}^{\prime}, u_{2 n}^{\prime}, u_{2 n}^{\prime}\right)+G\left(v_{2 n-1}^{\prime}, v_{2 n}^{\prime}, v_{2 n}^{\prime}\right)\right)} \varphi(t) d t .
\end{aligned}
$$

We have

$$
\begin{aligned}
\int_{0}^{G\left(u_{2 n}^{\prime}, u_{2 n+1}^{\prime}, u_{2 n+1}^{\prime}\right)} \varphi(t) d t & +\int_{0}^{G\left(v_{2 n}^{\prime}, v_{2 n+1}^{\prime}, v_{2 n+1}^{\prime}\right)} \varphi(t) d t \\
& \leq 8 k \int_{0}^{\left(G\left(u_{2 n-1}^{\prime}, u_{2 n}^{\prime}, u_{2 n}^{\prime}\right)+G\left(v_{2 n-1}^{\prime}, v_{2 n}^{\prime}, v_{2 n}^{\prime}\right)\right)} \varphi(t) d t
\end{aligned}
$$


holds for all $n \in N$. Thus, using (3.5) and (3.8) in (3.3), we get

$$
\begin{aligned}
\int_{0}^{G\left(u_{2 n+1}^{\prime}, u_{2 n+2}^{\prime}, u_{2 n+2}^{\prime}\right)} \varphi(t) d t & \leq k 8 k \int_{0}^{\left(G\left(u_{2 n-1}^{\prime}, u_{2 n}^{\prime}, u_{2 n}^{\prime}\right)+G\left(v_{2 n-1}^{\prime}, v_{2 n}^{\prime}, v_{2 n}^{\prime}\right)\right)} \varphi(t) d t \\
& \leq k(8 k)^{2} \int_{0}^{\left(G\left(u_{2 n-2}^{\prime}, u_{2 n-1}^{\prime}, u_{2 n-1}^{\prime}\right)+G\left(v_{2 n-2}^{\prime}, v_{2 n-1}^{\prime}, v_{2 n-1}^{\prime}\right)\right)} \varphi(t) d t \\
& \leq k(8 k)^{2 n} \int_{0}^{\left(G\left(u_{0}^{\prime}, u_{1}^{\prime}, u_{1}^{\prime}\right)+G\left(v_{0}^{\prime}, v_{1}^{\prime}, v_{1}^{\prime}\right)\right)} \varphi(t) d t \\
& \vdots \\
& \leq(8 k)^{2 n+1} \int_{0}^{\left(G\left(u_{0}^{\prime}, u_{1}^{\prime}, u_{1}^{\prime}\right)+G\left(v_{0}^{\prime}, v_{1}^{\prime}, v_{1}^{\prime}\right)\right)} \varphi(t) d t,
\end{aligned}
$$

and also, using (3.5) and (3.8) in (3.6), we get

$$
\begin{aligned}
\int_{0}^{G\left(u_{2 n}^{\prime}, u_{2 n+1}^{\prime}, u_{2 n+1}^{\prime}\right)} \varphi(t) d t & \leq 4 k(8 k) \int_{0}^{\left(G\left(u_{2 n-2}^{\prime}, u_{2 n-1}^{\prime}, u_{2 n-2}^{\prime}\right)+G\left(v_{2 n-1}^{\prime}, v_{2 n-1}^{\prime}, v_{2 n-1}^{\prime}\right)\right)} \varphi(t) d t \\
& \vdots \\
& \leq(8 k)^{2 n} \int_{0}^{\left(G\left(u_{0}^{\prime}, u_{1}^{\prime}, u_{1}\right)+G\left(v_{0}^{\prime}, v_{1}^{\prime}, v_{1}^{\prime}\right)\right)} \varphi(t) d t .
\end{aligned}
$$

Thus for all $n \in N$, we have

$$
\int_{0}^{G\left(u_{n}^{\prime}, u_{n+1}^{\prime}, u_{n+1}^{\prime}\right)} \varphi(t) d t \leq(8 k)^{n} \int_{0}^{\left(G\left(u_{0}^{\prime}, u_{1}^{\prime}, u_{1}^{\prime}\right)+G\left(v_{0}^{\prime}, v_{1}^{\prime}, v_{1}^{\prime}\right)\right)} \varphi(t) d t .
$$

Let $m, n \in N$ with $m>n$, we have

$$
\begin{aligned}
\int_{0}^{G\left(u_{n}^{\prime}, u_{m}^{\prime}, u_{m}^{\prime}\right)} \varphi(t) d t & \leq \int_{0}^{G\left(u_{n}^{\prime}, u_{n+1}^{\prime}, u_{n+1}^{\prime}\right)} \varphi(t) d t+\int_{0}^{G\left(u_{n+1}^{\prime}, u_{n+2}^{\prime}, u_{n+2}^{\prime}\right)} \varphi(t) d t \\
& +\cdots+\int_{0}^{G\left(u_{m-1}^{\prime}, u_{m}^{\prime}, u_{m}^{\prime}\right)} \varphi(t) d t .
\end{aligned}
$$

Since $8 k<1$, we get

$$
\begin{aligned}
\int_{0}^{G\left(u_{n}^{\prime}, u_{m}^{\prime}, u_{m}^{\prime}\right)} \varphi(t) d t & \leq \sum_{i=n}^{m-1}(8 k)^{i} \int_{0}^{\left(G\left(u_{0}^{\prime}, u_{1}^{\prime}, u_{1}^{\prime}\right)+G\left(v_{0}^{\prime}, v_{1}^{\prime}, v_{1}^{\prime}\right)\right)} \varphi(t) d t \\
& \leq \frac{(8 k)^{n}}{(1-8 k)} \int_{0}^{\left(G\left(u_{0}^{\prime}, u_{1}^{\prime}, u_{1}^{\prime}\right)+G\left(v_{0}^{\prime}, v_{1}^{\prime}, v_{1}^{\prime}\right)\right)} \varphi(t) d t
\end{aligned}
$$

We have

$$
\lim _{n, m \rightarrow+\infty} G\left(u_{n}^{\prime}, u_{m}^{\prime}, u_{m}^{\prime}\right)=0 .
$$

Thus $\left(u_{n}^{\prime}\right)$ is G-Cauchy in $g(Y)$. As $g(Y)$ is G-complete then subsequence $\left(u_{2 n+1}^{\prime}\right)=$ $\left(g a_{2 n+1}\right)$ and $\left(v_{2 n+1}^{\prime}\right)=\left(g b_{2 n+1}\right)$ are convergent to some $a \in Y$ and $b \in Y$ respectively. As we know that every sequence and subsequence of a G-Cauchy sequence are convergent to the same point. Hence $\left(u_{2 n}^{\prime}\right)=\left(h a_{2 n}\right)$ and $\left(v_{2 n}^{\prime}\right)=\left(h b_{2 n}\right)$ are also convergent. Since $g$ and $h$ are G-continuous, we have

$$
\left(g g a_{2 n+1}\right) \rightarrow g a,\left(h g a_{2 n+1}\right) \rightarrow h a,\left(g h a_{2 n}\right) \rightarrow g a,\left(h h a_{2 n}\right) \rightarrow h a
$$


and

$$
\left(g g b_{2 n+1}\right) \rightarrow g b,\left(h g b_{2 n+1}\right) \rightarrow h b,\left(g h b_{2 n}\right) \rightarrow g b,\left(h h b_{2 n}\right) \rightarrow h b .
$$

Since pairs $\{F, h\}$ and $\{S, g\}$ are commutative mappings, we have

$$
h g a_{2 n+1}=h F\left(a_{2 n}, b_{2 n}\right)=F\left(h a_{2 n}, h b_{2 n}\right)
$$

and

$$
g h a_{2 n}=g S\left(a_{2 n-1}, b_{2 n-1}\right)=S\left(g a_{2 n-1}, g a_{2 n-1}\right) .
$$

Thus

$$
\begin{aligned}
\int_{0}^{G\left(h g a_{2 n+1}, g h a_{2 n}, g h a_{2 n}\right)} \varphi(t) d t & =\int_{0}^{G\left(F\left(h a_{2 n}, h b_{2 n}\right), S\left(g a_{2 n-1}, g b_{2 n-1}\right), S\left(g a_{2 n-1}, g b_{2 n-1}\right)\right)} \varphi(t) d t \\
& \leq k \int_{0}^{\left(G\left(h h a_{2 n}, g g a_{2 n-1}, g g a_{2 n-1}\right)+G\left(h h b_{2 n}, g g b_{2 n-1}, g g b_{2 n-1}\right)\right)} \varphi(t) d t .
\end{aligned}
$$

Letting $n \rightarrow+\infty$, we have

$$
\int_{0}^{G(h a, g a, g a)} \varphi(t) d t=k \int_{0}^{(G(h a, g a, g a)+G(h b, g b, g b))} \varphi(t) d t .
$$

In the same way, we can show that

$$
\int_{0}^{G(h b, g b, g b)} \varphi(t) d t=k \int_{0}^{(G(h b, g b, g b)+G(h a, g a, g a))} \varphi(t) d t .
$$

Thus

$$
\int_{0}^{G(h a, g a, g a)} \varphi(t) d t+\int_{0}^{G(h b, g b, g b)} \varphi(t) d t=2 k \int_{0}^{(G(h a, g a, g a)+G(h b, g b, g b))} \varphi(t) d t .
$$

Since $2 k<8 k<1$, the last equality happens only if

$$
\int_{0}^{G(h a, g a, g a)} \varphi(t) d t=\int_{0}^{G(h b, g b, g b)} \varphi(t) d t=0 .
$$

Hence $h a=g a$ and $h b=g b$. Again

$$
\begin{aligned}
\int_{0}^{G\left(h g a_{2 n+1}, S(a, b), S(a, b)\right)} \varphi(t) d t & =\int_{0}^{G\left(F\left(h a_{2 n}, h b_{2 n}\right), S(a, b), S(a, b)\right)} \varphi(t) d t \\
& \leq k \int_{0}^{\left(G\left(h h a_{2 n}, g b, g b\right)+G\left(h h b_{2 n}, g b, g b\right)\right)} \varphi(t) d t .
\end{aligned}
$$

Letting $n \rightarrow+\infty$, we have

$$
\int_{0}^{G(h a, S(a, b), S(a, b))} \varphi(t) d t \leq k \int_{0}^{(G(h a, g b, g b)+G(h b, g b, g b))} \varphi(t) d t=0 .
$$

Thus, we get

$$
\int_{0}^{G(h a, S(a, b), S(a, b))} \varphi(t) d t=0 .
$$


Which implies that $S(a, b)=h a$. Similarly we can show that $S(b, a)=h b$. By using the same technique, we get

$$
\begin{aligned}
\int_{0}^{\left.G\left(F(a, b), g h a_{2 n}, g h a_{2 n}\right)\right)} \varphi(t) d t & =\int_{0}^{G\left(F(a, b), S\left(g a_{2 n-1}, g b_{2 n-1}\right), S\left(g a_{2 n-1}, g b_{2 n-1}\right)\right)} \varphi(t) d t \\
& \leq k \int_{0}^{\left(G\left(h a, g g a_{2 n-1}, g g a_{2 n-1}\right)+G\left(h b, g g b_{2 n-1}, g g b_{2 n-1}\right)\right)} \varphi(t) d t .
\end{aligned}
$$

Letting $n \rightarrow+\infty$, we have

$$
\int_{0}^{G(F(a, b), g a, g a))} \varphi(t) d t=k \int_{0}^{(G(h a, g a, g a)+G(h b, g b, g b))} \varphi(t) d t=0 .
$$

Thus, we get

$$
\int_{0}^{G(F(a, b), g a, g a))} \varphi(t) d t=0,
$$

which means that $F(a, b)=g a$. By using the same method we can show that $F(b, a)=h b$. Hence we get $g a=h a, g b=h b$ and $F(a, b)=g b, S(a, b)=h a, S(b, a)=h b$, by using Lemma 3.1 we have

$F(a, b)=g a=g b=F(b, a)=S(a, b)=h a=h b=S(b, a)$.

Now

$$
\begin{aligned}
\int_{0}^{G\left(g a_{2 n+1}, g a, g a\right)} \varphi(t) d t & =\int_{0}^{G\left(F\left(a_{2 n}, b_{2 n}\right), S(a, b), S(a, b)\right)} \varphi(t) d t \\
& \leq k \int_{0}^{\left(G\left(h a_{2 n}, g a, g a\right)+G\left(h b_{2 n}, g b, g b\right)\right)} \varphi(t) d t .
\end{aligned}
$$

Letting $n \rightarrow \infty$, we have,

$$
\int_{0}^{G(a, g a, g a)} \varphi(t) d t=k \int_{0}^{(G(a, g a, g a)+G(b, g b, g b))} \varphi(t) d t .
$$

Similarly, we can show that

$$
\int_{0}^{G(b, g b, g b)} \varphi(t) d t=k \int_{0}^{(G(b, g b, g b)+G(a, g a, g a))} \varphi(t) d t .
$$

Thus

$$
\int_{0}^{G(a, g a, g a)} \varphi(t) d t+\int_{0}^{G(b, g b, g b)} \varphi(t) d t=2 k \int_{0}^{(G(a, g a, g a)+G(b, g b, g b))} \varphi(t) d t .
$$

Since $2 k<8 k<1$, the last equality happens only if

$$
\int_{0}^{G(a, g a, g a)} \varphi(t) d t=\int_{0}^{G(b, g b, g b)} \varphi(t) d t=0 .
$$

Hence $a=g a$ and $b=g b$. Thus, we get

$F(a, a)=S(a, a)=g a=h a=a$.

For uniqueness, let $y \in Y$ with $y \neq a$ such that

$F(y, y)=S(y, y)=g y=y$. 
Then

$$
\begin{aligned}
\int_{0}^{G(a, y, y)} \varphi(t) d t & =\int_{0}^{G(F(a, a), S(y, y), S(y, y))} \varphi(t) d t \\
& \leq k \int_{0}^{G(h a, g y, g y)+G(h a, g y, g y)} \varphi(t) d t \\
& =k \int_{0}^{G(a, y, y)+G(a, y, y)} \varphi(t) d t \\
& =2 k \int_{0}^{G(a, y, y)} \varphi(t) d t .
\end{aligned}
$$

Since $2 k<8 k<1$, we get

$$
\int_{0}^{G(a, y, y)} \varphi(t) d t<\int_{0}^{G(a, y, y)} \varphi(t) d t
$$

Which is a contradiction. Thus $F, S, g, h$ have a unique common fixed point.

3.2. Corollary. Let $(Y, G)$ be a G-metric space. Let $F, S: Y \times Y \rightarrow Y$ and $g, h: Y \rightarrow Y$ be two mappings such that

$$
\int_{0}^{G(F(a, b), S(p, q), S(p, q))} \varphi(t) d t \leq k \int_{0}^{(G(h a, g p, g p)+G(h b, g q, g q))} \varphi(t) d t
$$

for all $a, b, p, q \in Y$ and $\varphi:[0,+\infty) \rightarrow[0,+\infty)$ is a Lebesgue integrable mapping which is summable such that for each $\epsilon>0, \int_{0}^{\epsilon} \varphi(t) d t>0$. Assume that $F, S$ and $g, h$ satisfy the following conditions:

(i) $F(Y \times Y) \subset g(Y) S(Y \times Y) \subset h(Y)$

(ii) $g(Y)$ or $h(Y)$ is complete, and

(iii) $g$ and $h$ is G-continuous and pairs $\{F, h\}$ and $\{S, g\}$ are of commuting mappings.

If $k \in\left[0, \frac{1}{8}\right)$, then there is a unique $a \in Y$ such that $F(a, a)=S(a, a)=g(a)=h(a)=a$.

Proof. In Theorem 3.1 by taking $c=p$ and $q=r$.

3.3. Example. Let $Y=[0,1]$. Define $G: Y \times Y \times Y \rightarrow R^{+}$by $G(a, b, c)=|a-b|+|a-c|+|b-c|$ for all $a, b, c \in Y$. Then $(Y, G)$ is a complete $\mathrm{G}$-metric space. Define mappings $F, S: Y \times Y \rightarrow Y$ and $g, h: Y \rightarrow Y$ by

$$
F(a, b)=\frac{1}{36} a b, S(a, b)=\frac{1}{144} a b \text { and } g a=\frac{1}{4} a, h a=\frac{1}{2} a .
$$

Since $|a b-p q|=|a-p|+|b-q|$ holds for all $a, b, p, q \in Y$.

Then the condition of Theorem (3.1) holds, in fact

$$
\begin{aligned}
\int_{0}^{G(F(a, b), S(p, q), S(c, r))} \varphi(t) d t & =\int_{0}^{\left(\left|\frac{1}{36} a b-\frac{1}{144} p q\right|+\left|\frac{1}{144} p q-\frac{1}{144} c r\right|+\left|\frac{1}{144} c r-\frac{1}{36} a b\right|\right)} \varphi(t) d t \\
& \leq \frac{1}{9} \int_{0}^{\left\{\left|\frac{1}{2} a-\frac{1}{4} p\right|+\left|\frac{1}{4} p-\frac{1}{4} c\right|+\left|\frac{1}{4} c-\frac{1}{2} a\right|+\left|\frac{1}{2} b-\frac{1}{4} q\right|+\left|\frac{1}{4} q-\frac{1}{4} r\right|+\left|\frac{1}{4} r-\frac{1}{2} b\right|\right\}} \varphi(t) d t \\
& =\frac{1}{9} \int_{0}^{G(h a, g p, g c)+G(h b, g q, g r)} \varphi(t) d t
\end{aligned}
$$

holds for all $a, b, c, p, q, r \in Y$. It is easy to see that $F, S, g, h$ satisfies all the hypothesis of Theorem 3.1. Thus $F, S, g, h$ have a unique common fixed point. Here $F(0,0)=$ $S(0,0)=g 0=h 0=0$. 


\section{References}

[1] M. Abbas, G. Jungck, Common fixed point results for noncommuting mappings without continuity in cone metric spaces, J. Math. Anal. Appl., Vol. 341, 2008, pp. 416-420.

[2] M. Abbas, B. E. Rhoades, Common fixed point results for noncommuting mapping without continuity in generalized metric spaces, Applied Mathematics and Computation, Vol. 215, 2009, pp. 262-269.

[3] Th. Abdeljawad, Completion of cone metric spaces, Hacet. J. Math. Stat., Vol. 39, 2010, pp. 67-74.

[4] I. Beg, M. Abbas, Coincidence point and invariant approximation for mappings satisfying generalized weak contractive condition, Fixed Point Theory. Appl., Article ID 74503, 2006, pp. 1-7.

[5] T. G. Bhaskar, G. V. Lakshmikantham, Fixed point theorems in partially ordered metric spaces and applications, Nonlinear Analysis, Vol. 65, 2006, pp. 1379-1393.

[6] A. Branciari, A fixed point theorem for mappings satisfying a general contractive condition of integral type, International Journal of Mathematics and Mathematical Sciences, Vol. 29, No. 9, 2002, pp. 531-536.

[7] L. G. Haung, X. Zhang, Cone metric spaces and fixed point theorems of contractive mappings, J. Math. Anal. Appl., Vol. 332, 2007, pp. 1468-1476.

[8] G. Jungck, Commuting maps and fixed points, Am. Math. Monthly., Vol. 83, 1976, pp. 261-263.

[9] G. Jungck, Compatible mappings and common fixed points, Int. J. Math. Sci., Vol. 9 (4), 1986, pp. 771-779.

[10] G. Jungck, Common fixed points for commuting and compatible maps on compacta, Proc. Am. Math. Soc., Vol. 103, 1988, pp. 977-983.

[11] G. Jungck, N. Hussain, Compatible maps and invariant approximations, J. Math. Anal. Appl., Vol. 325 (2), 2007, pp. 1003-1012.

[12] F. Khojasteh, Z. Goodarzi, A. Razani, Some Fixed Point Theorems of Integral Type Contraction in Cone Metric Spaces, Fixed Point Theory Appl., Vol. 2010, Article ID 189684, 13 pages, 2010.

[13] V. Lakshmikantham, Lj. B. Cirić, Coupled fixed point theorems for nonlinear contractions in partially ordered metric spaces, Nonlinear Analysis, Vol. 70, 2009, pp. 4341-4349.

[14] K. Manish, Coupled coincidence fixed point theorem for two pairs in G-metric spaces, Int. J. Math. Appl., vol. 3, 2014, pp. 53-62.

[15] P. P. Murthy, K. Tas, New common fixed point theorems of Gregus type for R-weakly commuting mappings in 2-metric spaces, Hacet. J. Math. Stat., Vol. 38, 2009, pp. 285291.

[16] Z. Mustafa, B. Sims, A new approach to generalized metric spaces, J. Nonlinear Convex Anal., Vol. 7 (2), 2006, pp. 289-297.

[17] R. P. Pant, Common fixed points of noncommuting mappings, J. Math. Anal. Appl. Vol. 188, 1994, pp. 436-440.

[18] V. Popa, M. Mocanu, Altering distance and common fixed points under implicit rela tions, Hacet. J. Math. Stat., Vol. 38, 2009, pp. 329-337.

[19] W. Shatanawi, Coupled fixed point theorems in generalized metric spaces, Hecet. J. Math. Stat., Vol. 40 (3), 2011, pp. 441-447. 